\title{
A study of spatial structure of galactic open star clusters
}

\author{
Nilakshi $^{1}$, R. Sagar ${ }^{1,2}$, A. K. Pandey ${ }^{1}$, and V. Mohan ${ }^{1}$ \\ 1 State Observatory, Manora Peak, Nainital 263 129, India \\ 2 Indian Institute of Astrophysics, Bangalore 560 034, India
}

Received 14 May 2001 / Accepted 8 November 2001

\begin{abstract}
In order to study the relation between the core and corona in galactic star clusters, the spatial structure of 38 rich open star clusters has been studied using radial density profiles derived from the photometric data of the Digital Sky Survey. The shape of the radial density profile indicates that the corona, most probably, is the outer region around the cluster. It can exist from the very beginning of the cluster formation and dynamical evolution is not the reason for its occurrence. The study does not find any relation between cluster size and age but indicates that the clusters with galacto-centric distances $>9.5 \mathrm{kpc}$ have larger sizes. Further, we find that the average value of the core radius is $1.3 \pm 0.7 \mathrm{pc}$ and that of annular width of the corona is $5.6 \pm 1.9 \mathrm{pc}$, while average values of densities of cluster members in the core and corona are $15.4 \pm 9.9 \mathrm{star} / \mathrm{pc}^{2}$ and $1.6 \pm 0.99 \mathrm{star} / \mathrm{pc}^{2}$ respectively. Average field star contaminations in the core and corona are $\sim 35 \%$ and $80 \%$ respectively. In spite of smaller densities in the coronal region, it contains $\sim 75 \%$ of the cluster members due to its larger area in comparison to the core region. This clearly demonstrates the importance of the coronal region in studies dealing with the entire stellar contents of open star clusters as well as their dynamical evolution. In contrast to the cluster cores, the structure of coronal regions differs significantly from one cluster to other.
\end{abstract}

Key words. galaxy: open clusters and associations: general - stellar content

\section{Introduction}

The stars of a stellar cluster are presumably the offspring of the same interstellar dust and clouds that are born together. Hence, they are dynamically associated and gravitationally bound and are located approximately at the same distance. A spatial structure represents how sibling stars are distributed within a family of stars (cluster) according to their mass or brightness. The study of the spatial structure of a star cluster therefore provides an opportunity to determine the mechanisms and conditions of cluster formation at different stages of a galactic evolution. At the beginning of the 20th century, Shapley (1916), pointed out that the dimensions of star clusters considerably exceed the sizes that are obtained directly by simple eye estimates. However, no detailed studies of outer regions of clusters were carried out until the 1960s. Artyukhina \& Kholopov (1959, 1963, 1966), Artyukhina (1966) and Kholopov (1969) suggested that all clusters, from very sparse ones like Coma Bernices to the richest of all the Galactic globular clusters have unity not only in origin and development (Elmegreen \& Efremov 1997) but also show unity in the structure and have come to believe that these extended regions represent very

Send offprint requests to: R. Sagar,

e-mail: sagar@upso.ernet.in important structural properties of the star clusters. Now, it is well established that every cluster consists of two main regions: (i) nucleus (or core) which is the densest central part of the system, the region that is perceived directly by the eye as a star cluster and (ii) halo (or corona ) which is an outer and extended region with lower density around a cluster, that contains a substantial number of cluster members.

Until recently most studies have been made of the nuclei which have been taken to represent the clusters as a whole. Extensive studies of the coronal regions have not been carried out mainly because of the non-availability of photometry in a large field around the star clusters. The study of stars in the coronal region is important for the following main reasons.

\subsection{Slope of initial mass function (IMF) towards low mass}

Wilner \& Lada (1991) observed the presence of dip in luminosity function at magnitudes $1.5<M_{v}<3.5$ and they give two possible explanations for the observed deficit of low luminosity stars in NGC 2362: (i) a significant populations of low luminosity cluster stars are present in the outer part of the cluster or (ii) the cluster IMF is different from Salpeter (1955) field star IMF in the vicinity of Sun. 


\subsection{Dynamical study of open clusters}

Until some years ago, unlike the coronal region of globular clusters, the coronal regions of open clusters did not attract sufficient attention although from a dynamical point of view they represent an exciting and challenging field of study. On the observational side, the challenge is to isolate the cluster members from the general field stars, as open star clusters $(\mathrm{OCls})$ are usually projected against rich stellar fields of the Galactic disk. On the theoretical side, open clusters are considered dynamically active systems. Unlike globular clusters and galaxies, where two body relaxation times are very much longer than the crossing times and the system can be considered to be in quasi-static equilibrium, the crossing and relaxation times of open clusters are more commensurate with each other. Thus the evolution of clusters, velocity-distribution due to stellar encounters cannot be treated independently of the orbital mixing of the distribution. It is thus essential to study the dynamics of small number $(N)$ stellar systems like the open clusters as compared to large $N$ systems because any differences in their velocity distributions that occur due to the close stellar encounters with clouds in the interstellar medium or passing molecular clouds or the effect of galactic tidal field will appear primarily in the high energy tails of the distributions.

\subsection{Cluster membership for peculiar stars}

Turner (1982) recommend that it is necessary to fully delineate the boundaries of the cluster nucleus and corona to strengthen the case for the cluster membership of Cepheid variables e.g. of V Cent. in NGC 5662.

The above discussions clearly indicate that a systematic study of the spatial stellar distribution in OCls would be desirable and the same has been presented here. The criteria used to select the clusters for the present study are described in the next section.

\section{Selection criteria}

If existence of the corona observed in the open clusters is a reality then it is important to know whether the corona is due to the dynamical evolution or is an imprint of the star formation process itself. This question may be better understood by studying and comparing the OCls of all ages, very young to extremely old. Another significant aspect to be addressed is to determine if the existence of a corona in an open cluster is position-dependent in the Galaxy. In order to answer these questions, one would like to consider the OCls situated not only in different locations of the Galaxy but also having a statistically significant number of stars for the study of coronal regions. We have therefore selected only rich clusters from the open clusters' catalogue (Lyngå 1987) covering a range in age and galactic position, except the binary and multiple clusters. We exclude them because their proximity tends to influence each others dynamics which makes the determination of their individual radial density profiles (RDPs) $\rho(R)$ unreliable. If there is more than one cluster in the $1^{\circ} \times 1^{\circ}$ area around the cluster in consideration, they are treated as binary/multiple clusters and hence, not considered for the study. Some of the clusters excluded in this way are indeed found to be binary cluster e.g. NGC 869, NGC 884 and NGC 1912 (Subramaniam et al. 1995). We have also excluded some clusters whose $\rho(R)$ can not be used in the present study due to the limitations discussed below.

\subsection{Limitations of $R D P s$}

To obtain the radius using RDPs is an arduous task. It has limitations such as:

1. dependency of $\rho(R)$ on the limiting magnitude of the members. The more fainter stars considered, the larger the cluster radius, because such stars occupy larger distances from the cluster center due to two body relaxation. We have derived $\rho(R)$ using the stars up to an absolute magnitude of $M_{B}=+4$;

2. in some cases, the angular size is difficult to determine accurately due to the weak contrast between cluster and field stars in the outer region;

3. sometimes the irregular shape of the cluster also makes it difficult to accurately determine its boundaries;

4. the number of members diminishes with increasing distance from the cluster center and it becomes difficult to detect them in the statistical fluctuations of the field stars;

5 . in the case of very sparse and poor clusters or in the clusters that contain stars in clumps, it becomes difficult to locate the center.

Despite all these limitations, the method of RDP can still be used to study the structure of rich galactic clusters. On the basis of the above considerations, we found only 38 OCls in Lyngå's (1987) catalogue as potential candidates for the present study and general information about them is given in Table 1. The clusters are distributed in the Galactic longitude along the Galactic plane representing different locations in the Galaxy. They cover a range in ages from $\sim 10 \mathrm{Myr}$ to $8 \mathrm{Gyr}$; in visual angular diameter from about $3^{\prime}$ to $35^{\prime}$ and in distance from $0.9 \mathrm{kpc}$ to $2.6 \mathrm{kpc}$. The method of RDP determination and its use to determine the core and coronal regions, is described in the next section while the statistical properties and other results are discussed in the remaining part of the paper.

\section{Determination of radial density profile}

To cope with problems like interpreting the clusters' distinctive spatial distribution, homogeneous data on the parameters of OCls are essential. Accordingly, it is vital to adopt a definite instrumental system as well as a common approach for studying the size and structure of all OCls. Our aim is therefore also to include the outer region of the sample clusters. For this, the photoelectric, photographic 
Table 1. General information about the clusters under study with their sequence and IAU names. Galactic longitude $(l)$ and latitude $(b)$ in degrees, diameter $(D)$ in arcmin and Trumpler concentration classification are taken form Lyngå's (1987) catalogue while distance $(d)$ in pc and $\log (t)$ where $t$ is the age in years are borrowed from Loktin et al. (1994) catalogue, if available. Otherwise (asterisked clusters) they are taken from Lyngå's (1987) catalogue.

\begin{tabular}{|c|c|c|c|c|c|c|c|}
\hline Name & IAU name & $l$ & $b$ & Trumpler & $D$ & $d$ & $\log (t)$ \\
\hline NGC 188 & $\mathrm{C} 0039+850$ & 122.8 & 22.5 & $\mathrm{I} 2 \mathrm{r}$ & 13 & 1675 & 9.8 \\
\hline NGC 654 & $\mathrm{C} 0140+616$ & 129.1 & -0.3 & II $2 \mathrm{r}$ & 5 & 2422 & 7.1 \\
\hline NGC 663 & $\mathrm{C} 0142+610$ & 129.5 & -0.9 & II $3 r$ & 16 & 2284 & 7.1 \\
\hline NGC 1245 & $\mathrm{C} 0311+470$ & 146.6 & -8.9 & II $2 \mathrm{r}$ & 10 & 2608 & 9.2 \\
\hline NGC 1817 & $\mathrm{C} 0509+166$ & 186.1 & -13.1 & IV $2 \mathrm{r}$ & 15 & 2132 & 8.9 \\
\hline NGC 1960 & C0532+341 & 174.5 & 1.0 & I3r & 12 & 1285 & 7.5 \\
\hline NGC 2099 & $\mathrm{C} 0549+325$ & 177.6 & 3.1 & $\mathrm{I} 2 \mathrm{r}$ & 23 & 1356 & 8.5 \\
\hline NGC 2244 & $\mathrm{C} 0629+049$ & 206.4 & -2.0 & II3r & 23 & 1640 & 6.8 \\
\hline Trumpler $5^{*}$ & $\mathrm{C} 0634+094$ & 202.9 & 1.0 & III1r & 7 & 977 & 9.9 \\
\hline NGC 2301 & C0649+005 & 212.6 & 0.3 & $\mathrm{I} 3 \mathrm{r}$ & 12 & 857 & 8.2 \\
\hline NGC 2323 & $\mathrm{C} 0700-082$ & 221.7 & -1.2 & II3r & 16 & 997 & 8.0 \\
\hline NGC 2345 & C0706 - 130 & 226.6 & -2.3 & II3r & 12 & 1947 & 7.6 \\
\hline NGC 2354 & $\mathrm{C} 0712-256$ & 238.4 & -6.8 & III $2 r$ & 20 & 2089 & 8.8 \\
\hline NGC 2360 & $\mathrm{C} 0715-155$ & 229.8 & -1.4 & $\mathrm{I} 3 \mathrm{r}$ & 12 & 1459 & 9.0 \\
\hline NGC 2420 & $\mathrm{C} 0735+216$ & 198.1 & 19.6 & I1r & 10 & 2464 & 9.3 \\
\hline NGC 2421 & $\mathrm{C} 0734-205$ & 236.2 & 0.1 & $\mathrm{I} 2 \mathrm{r}$ & 10 & 2001 & 7.2 \\
\hline NGC 2437 & C0739 - 147 & 231.9 & 4.1 & II $2 r$ & 27 & 1491 & 8.4 \\
\hline NGC 2447 & C0742 - 237 & 240.1 & 0.2 & I3r & 22 & 1014 & 8.7 \\
\hline NGC 2477 & $\mathrm{C} 0750-384$ & 253.6 & -5.8 & $\mathrm{I} 2 \mathrm{r}$ & 27 & 1796 & 8.9 \\
\hline NGC 2533 & C0805- 297 & 247.8 & 1.3 & II $2 \mathrm{r}$ & 3 & 1551 & 9.3 \\
\hline NGC 3114 & C1001 - 598 & 283.3 & -3.8 & II3r & 35 & 893 & 7.9 \\
\hline IC $2714^{*}$ & C1115-624 & 292.4 & -1.8 & II $2 r$ & 12 & 1138 & 8.3 \\
\hline Mel 105 & C1117-632 & 292.9 & -2.5 & $\mathrm{I} 2 \mathrm{r}$ & 4 & 2035 & 8.3 \\
\hline NGC 4755 & $\mathrm{C} 1250-600$ & 303.2 & 2.5 & $\mathrm{I} 3 \mathrm{r}$ & 10 & 2105 & 7.3 \\
\hline NGC 5617 & $\mathrm{C} 1426-605$ & 314.7 & -0.1 & I3r & 10 & 1537 & 7.6 \\
\hline NGC 6208 & $\mathrm{C} 1645-537$ & 333.7 & -5.8 & III $2 \mathrm{r}$ & 15 & 1248 & 9.3 \\
\hline NGC 6250 & C1654 - 457 & 340.8 & -1.8 & II $3 \mathrm{r}$ & 7 & 942 & 7.3 \\
\hline NGC 6451 & $\mathrm{C} 1747-302$ & 359.5 & -1.6 & $\mathrm{I} 2 \mathrm{r}$ & 7 & 1915 & 8.1 \\
\hline NGC 6531 & C1801 - 225 & 7.7 & -0.4 & $\mathrm{I} 3 \mathrm{r}$ & 13 & 1285 & 7.0 \\
\hline NGC 6546 & C1804 - 233 & 7.3 & -1.4 & II1r & 13 & 927 & 7.9 \\
\hline NGC 6705 & $\mathrm{C} 1848-063$ & 27.3 & -2.8 & $\mathrm{I} 2 \mathrm{r}$ & 13 & 1794 & 8.3 \\
\hline NGC 6755 & $\mathrm{C} 1905+041$ & 38.5 & -1.7 & $\mathrm{II} 2 \mathrm{r}$ & 14 & 1563 & 7.8 \\
\hline NGC 6811 & $\mathrm{C} 1936+464$ & 79.4 & 11.9 & III1r & 12 & 1292 & 8.8 \\
\hline NGC 6819 & $\mathrm{C} 1939+400$ & 74.0 & 8.5 & $\mathrm{I} 1 \mathrm{r}$ & 5 & 1689 & 9.4 \\
\hline NGC 6866 & $\mathrm{C} 2002+438$ & 79.5 & 6.9 & II $2 r$ & 6 & 1490 & 8.8 \\
\hline NGC $6939^{*}$ & $\mathrm{C} 2030+604$ & 95.9 & 12.3 & II1r & 7 & 1202 & 9.2 \\
\hline NGC 6940 & $\mathrm{C} 2032+281$ & 69.9 & -7.2 & III $2 \mathrm{r}$ & 31 & 1042 & 8.9 \\
\hline NGC 7654 & $\mathrm{C} 2322+613$ & 112.8 & 0.5 & II $2 \mathrm{r}$ & 12 & 1490 & 7.7 \\
\hline
\end{tabular}

and most of the CCD photometric data published so far are not sufficient as most of them are limited to the core regions only. We therefore used the photometric results published in the U.S. Naval Observatory (USNO)-A V2.0 catalogue (Monet et al. 1998). The data are derived from the images which are Digitized Schmidt Survey (DSS) plates covering the entire sky. Northern hemisphere images are constructed from Palomar Sky Survey while those for Southern are from UK Science and Engineering Research Council sky survey. Although the photometric quality of the USNO catalogue data is inferior (see Appendix) to photoelectric and CCD, it is good enough to obtain cluster structural parameters. The clusters showing indication of well defined corona are planned for further extensive study using a large format CCD camera. In fact,
Pandey et al. (2001) and Nilakshi \& Sagar (2002) have already carried out such a study for our sample clusters NGC 7654 and NGC 2099 respectively.

\subsection{Spatial coverage of the photometric data}

In order to sketch the RDP well beyond the outer region of the sample clusters whose angular diameter is less than $20^{\prime}$ in Lyngå's (1987) catalogue, we extract a circular area of $1^{\circ}$ diameter centered on the cluster from the USNO CDROMs. However, for the clusters having diameter $>20^{\prime}$, the extracted area is of $2^{\circ}$ diameter. Thus, we determine the RDP far beyond the cluster size and certainly include the field region. 


\subsection{Center of cluster}

To outline the RDPs, the first necessary step is to identify the cluster center. We derive it (cf. Sagar \& Griffiths 1998) iteratively by calculating average $X$ and $Y$ position of stars within $2^{\prime}$ or $3^{\prime}$ around an eye-estimated center until it converged to a constant value. An error of few tens of arcsec is expected in locating the cluster center.

\subsection{Stellar surface density}

For determining the RDP of stars around a cluster region, the extracted area has been divided into a number of concentric circles with respect to the above estimated cluster-center in such a way that each zone contains a statistically significant number of stars (generally more than 20). An annular width of $\sim 1^{\prime}$ has been used for all the clusters under study. The stellar surface density, $\rho_{i}$, in the $i$ th zone is defined as:

$\rho_{i}=\frac{N_{i}}{A_{i}}=\frac{N_{i}}{\pi\left(R_{i}^{2}-R_{i-1}^{2}\right)} ;$

where $N_{i}$ is the number of stars up to absolute magnitude $M_{B}=+4$ in the the area $A_{i}$ of the $i$ th annulus whose outer and inner radii are $R_{i}$ and $R_{i-1}$ respectively. The RDPs derived in this way are shown in Fig. 1 for all the clusters under study. The error bars are calculated assuming that the number of stars in a zone is governed by Poisson statistics. Following Kaluzny (1992), we describe the $\rho(R)$ of an open star cluster as:

$\rho(R) \propto \frac{f_{0}}{1+\left(\frac{R}{R_{0}}\right)^{2}} ;$

where the cluster core radius $R_{0}$ is the radial distance at which the value of $\rho(R)$ becomes half of the central density $f_{0}$. We fit this function to the observed data points of each cluster and use $\chi^{2}$ minimization technique to determine $R_{0}$ and other constants. As can be seen in Fig. 1, the fitting of the function is satisfactory except for the clusters NGC 1817, NGC 6451 and NGC 6940. The radial distance $\left(R_{\mathrm{cn}}\right)$ at which the $\rho(R)$ becomes constant is defined as the extent of the cluster. These angular dimensions have been converted to linear ones using values of the cluster distances given in Table 1. The linear sizes derived in this way are listed in Table 2 along with other parameters obtained from RDPs. The error in $R_{0}$ is derived from the $\chi^{2}$ fitting. We expect $\sim 10 \%$ error in the estimation of $R_{\mathrm{cn}}$.

\section{The structural properties of the clusters}

The mean stellar densities $\rho_{0}=\frac{N_{0}}{\pi R_{0}^{2}}$ and $\rho_{\mathrm{cn}}=\frac{N_{\mathrm{cn}}}{\pi\left(R_{\mathrm{cn}}^{2}-R_{0}^{2}\right)}$ given in Table 2 are for the core and coronal regions respectively where $N_{0}$ and $N_{\text {cn }}$ are the number of stars in the corresponding regions. Assuming $\rho_{\mathrm{f}}$ as the field star density, the densities of cluster members in the core and coronal regions are $\left(\rho_{0}-\rho_{\mathrm{f}}\right)$ and $\left(\rho_{\mathrm{cn}}-\rho_{\mathrm{f}}\right)$ respectively.
We estimate $\rho_{\mathrm{f}}$ at the cluster distance from its surrounding regions and use it to evaluate the field star contaminations in the both core and coronal regions along with the fraction of cluster members in the corona. The values thus derived are given in Table 2. The field star density is minimum towards NGC 2420 with a value of $1.1 \mathrm{star} / \mathrm{pc}^{2}$ while maximum value of $26.1 \mathrm{star} / \mathrm{pc}^{2}$ is observed towards NGC 6451.

\subsection{Structure of stellar surface density distribution}

In order to see whether the $\rho(R)$ within a cluster radius follows $\rho \propto \mathrm{e}^{-B R}$ or $\rho \propto R^{-\gamma}$, we fit the linear relations $\ln (\rho)=$ const. $-B R$ and $\log (\rho)=$ const. $-\gamma \log (R)$ between $\rho$ and $R$, up to both the core and cluster radii. The slopes $B$ and $\gamma$ estimated using least-squares solution are listed in Table 3 along with their standard deviations $\sigma_{B}$ and $\sigma_{\gamma}$ and correlation coefficients $c_{1}$ and $c_{2}$ of the linear relations. As the values of $c_{1}$ and $c_{2}$ are generally of the same order, the RDP within a cluster radius can be represented by either $\rho \propto \mathrm{e}^{-B R}$ or $\rho \propto R^{-\gamma}$. However, in some clusters one relation seems to fit data points better than other one. The values of $B$ and $\gamma$ can be used to study differences between the structure of the core and corona of the clusters under study. In the core region, the values of $B$ change from 0.001 to 0.01 with a median of 0.003 . By including the coronal region, the value of $B$ decreases, as expected (see Fig. 1) by an average of 0.003 . On the other hand, in the core the values of $\gamma$ range from 0.12 to 0.72 with a median of 0.34 . In contrast to $B$, the value of $\gamma$ thus shows relatively larger variation and increases by including the coronal region. This is due only to the role of the constants in the relations under discussion. However, variation in both $B$ and $\gamma$ values by including the coronal region is not the same for all clusters, indicating that the shape of the coronal regions is different for different clusters, as can also be seen in Fig. 1. Differences in the structure of the corona have also been observed by Durgapal \& Pandey (2001) in a sample of 4 clusters. They may be due to various reasons e.g., (i) the dynamical relaxation which causes mass segregation in the cluster (Nilakshi \& Sagar 2002 and references therein), (ii) the external environment, as we know that the corona of the clusters are moulded by the Galactic tidal fields (Mathieu 1985).

\section{Properties of clusters core and coronal regions}

In order to obtain a general view of the real constitution of the galactic clusters, the frequency distributions and the correlation between some of the cluster parameters contained in Table 2 are studied in this section. As the number of sample clusters is only 38 , some of the conclusions drawn below may not be definitive but indicative, which may be worthwhile to study in detail when a larger sample becomes available. 


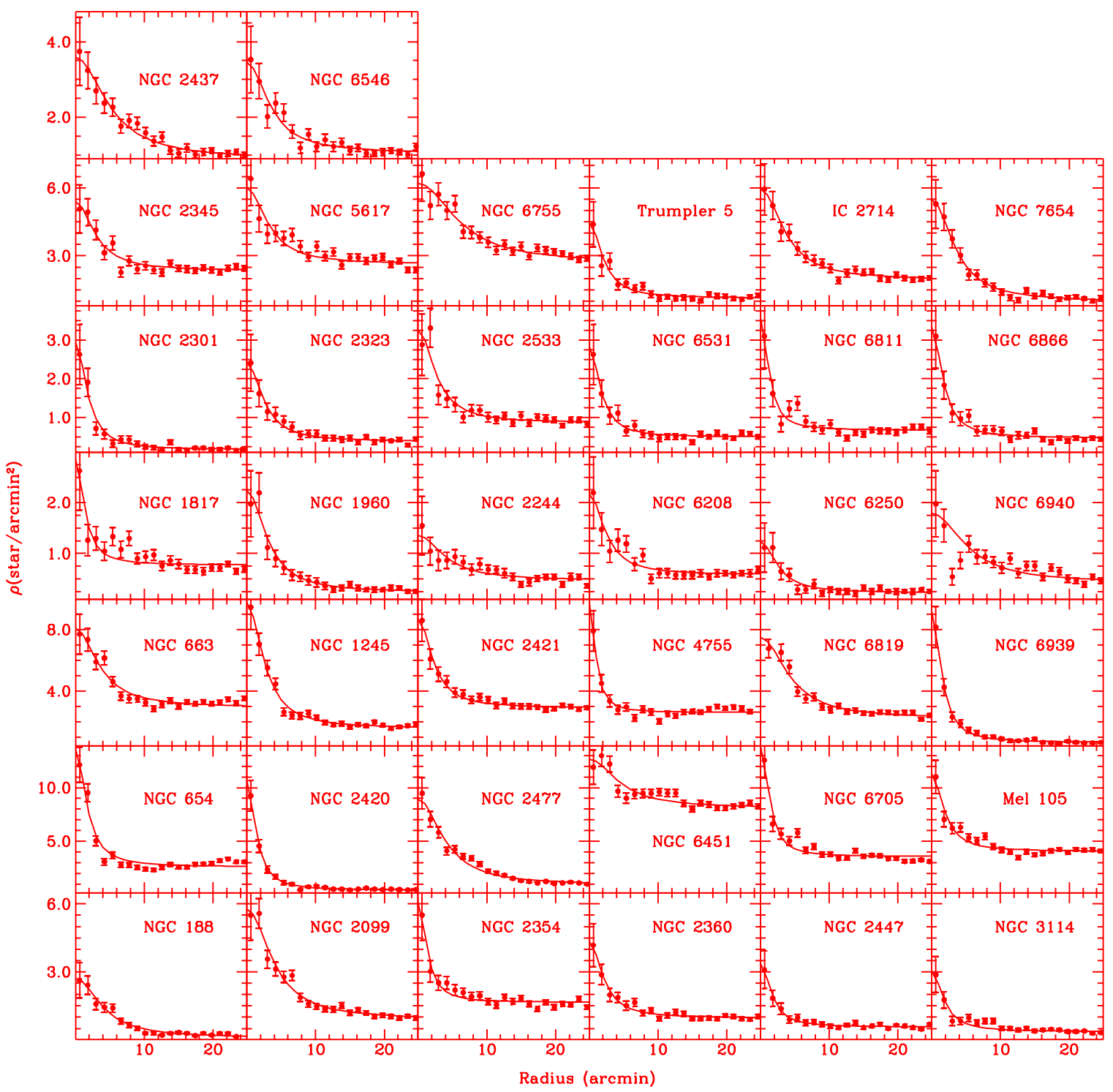

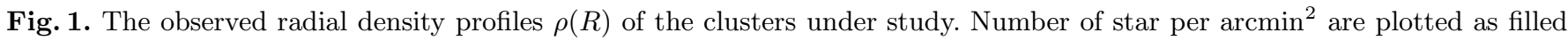
circles against the cluster radius in arcmin. The lengths of the bars represent errors due to sampling statistics. The continuous curve is the fitted function $\rho(R) \propto \frac{f_{0}}{1+\left(\frac{R}{R_{0}}\right)^{2}}$ to the observed points using $\chi^{2}$ minimization technique.

\subsection{Frequency distribution of cluster parameters}

Figure 2 illustrates the histograms of sizes, cluster member densities and field star contamination in the core and coronal regions along with fraction of cluster members in corona of clusters under study. The core size ranges from $\sim 0.5$ to 2.9 pc. Its histogram in Fig. 2 a has a well defined peak around $0.75 \mathrm{pc}$ with an average of $1.3 \pm 0.67 \mathrm{pc}$ and a median of $1.2 \mathrm{pc}$. In contrast, the histogram of width of the coronal region show a relatively flat distribution with values ranging from 2.5 to $9.3 \mathrm{pc}$. Its mean and median values are $5.6 \pm 1.9$ and $5.54 \mathrm{pc}$ respectively. The coronal width is thus more than 4 times the core size.

In Fig. 2b, histograms of the mean cluster member densities in the core $\left(\rho_{0}-\rho_{\mathrm{f}}\right)$ and corona $\left(\rho_{\mathrm{cn}}-\rho_{\mathrm{f}}\right)$ regions are displayed. They have a mean and a median of $15.4 \pm$ 9.9 and $13.2 \mathrm{star} / \mathrm{pc}^{2}$ respectively with large range from
0.6 to $53 \mathrm{star} / \mathrm{pc}^{2}$ in the core but relatively small range from 0.3 to $4.5 \mathrm{star} / \mathrm{pc}^{2}$ with a mean and a median of $1.6 \pm 0.99$ and $1.3 \mathrm{star} / \mathrm{pc}^{2}$ respectively in the corona. The density of cluster members in the corona has a sharp peak around 1 star $/ \mathrm{pc}^{2}$ while that in the core has extended distribution which peaks around $11 \mathrm{star} / \mathrm{pc}^{2}$. All these indicate that the density of cluster members in the core is about 10 times larger than that of the corona.

Figure 2c shows the histograms of field star contamination in both the core and coronal regions. The core region of NGC 1817 is least contaminated with a value of $6 \%$ while that of NGC 5617 has the maximum contamination of $60 \%$. The histogram for the core region peaks around $25 \%$ with a median value of $28 \%$. As expected, the field star contamination in the corona is more in comparison to the core. Its histogram has a peak around $80 \%$ with a median of $75 \%$. 
Table 2. Structural parameters of the clusters under study derived with the help of RDP. Column 1 denotes the cluster name; Col. 2 is the galacto-centric distance $\left(R_{\mathrm{G}}\right)$ in kpc (in converting distance from geo-centric to galacto-centric, $R_{\mathrm{G}}$ value for the Sun is taken as $8.5 \mathrm{kpc})$; Col. 3 is $z$, the height above or below the plane of our Galaxy; Cols. $4-6$ denote radius $\left(R_{0}\right)$, mean stellar density $\left(\rho_{0}\right)$ and percentage of field star contamination $\left(F_{0}\right)$ respectively for the core region while Cols. 7-9 represent the corresponding values for the coronal region. Annular width of the corona $\left(R_{\mathrm{cn}}-R_{0}\right)$ has an accuracy of $\sim 10 \%$. Column 10 denotes mean field star density $\left(\rho_{\mathrm{f}}\right)$ determined with an error of about $\pm 0.1 \mathrm{star} / \mathrm{pc}^{2}$. Densities of cluster members in the core and coronal regions are thus $\left(\rho_{0}-\rho_{\mathrm{f}}\right)$ and $\left(\rho_{\mathrm{cn}}-\rho_{\mathrm{f}}\right)$ respectively. Last column presents the percentage of cluster members $\left(M_{\mathrm{cn}}\right)$ in the coronal region.

\begin{tabular}{|c|c|c|c|c|c|c|c|c|c|c|}
\hline \multirow[b]{2}{*}{ Name } & \multirow[b]{2}{*}{$\begin{array}{c}R_{\mathrm{G}} \\
(\mathrm{kpc})\end{array}$} & \multirow[b]{2}{*}{$\begin{array}{c}z \\
(\mathrm{pc})\end{array}$} & \multicolumn{3}{|c|}{ Core } & \multicolumn{3}{|c|}{ Corona } & \multirow[b]{2}{*}{$\begin{array}{c}\rho_{\mathrm{f}} \\
\left(\operatorname{star} / \mathrm{pc}^{2}\right)\end{array}$} & \multirow[b]{2}{*}{$\begin{array}{l}M_{\mathrm{cr}} \\
(\%)\end{array}$} \\
\hline & & & $\begin{array}{c}R_{0} \\
(\mathrm{pc})\end{array}$ & $\begin{array}{c}\rho_{0} \\
\left(\mathrm{star} / \mathrm{pc}^{2}\right)\end{array}$ & $\begin{array}{r}F_{0} \\
(\%)\end{array}$ & $\begin{array}{c}\left(R_{\mathrm{cn}}-R_{0}\right) \\
(\mathrm{pc})\end{array}$ & $\begin{array}{c}\rho_{\mathrm{cn}} \\
\left(\mathrm{star} / \mathrm{pc}^{2}\right)\end{array}$ & $\begin{array}{l}F_{\text {cn }} \\
(\%)\end{array}$ & & \\
\hline NGC 188 & 9.5 & 641.0 & $2.05 \pm 0.02$ & $7.9 \pm 0.8$ & 15 & 4.45 & $2.1 \pm 0.3$ & 44 & 1.2 & 57 \\
\hline NGC 654 & 10.2 & -12.7 & $1.26 \pm 0.14$ & $23.3 \pm 2.1$ & 22 & 5.79 & $6.5 \pm 0.2$ & 74 & 5.3 & 67 \\
\hline NGC 663 & 10.1 & -35.9 & $2.45 \pm 0.29$ & $15.0 \pm 0.9$ & 47 & 6.41 & $8.0 \pm 0.2$ & 83 & 7.1 & 58 \\
\hline NGC 1245 & 10.8 & -403.5 & $2.06 \pm 0.10$ & $12.9 \pm 1.0$ & 25 & 8.56 & $4.2 \pm 0.1$ & 70 & 3.1 & 73 \\
\hline NGC 1817 & 10.6 & -483.2 & $0.90 \pm 0.21$ & $6.1 \pm 0.7$ & 6 & 9.02 & $2.2 \pm 0.1$ & 75 & 1.9 & 58 \\
\hline NGC 1960 & 9.8 & 22.4 & $1.19 \pm 0.12$ & $12.4 \pm 1.7$ & 16 & 4.54 & $3.3 \pm 0.2$ & 57 & 2.1 & 70 \\
\hline NGC 2099 & 9.9 & 73.3 & $1.62 \pm 0.12$ & $27.3 \pm 1.8$ & 25 & 6.53 & $9.1 \pm 0.2$ & 70 & 6.9 & 73 \\
\hline NGC 2244 & 10.0 & -57.2 & $2.04 \pm 0.41$ & $4.1 \pm 0.6$ & 49 & 5.91 & $2.7 \pm 0.1$ & 72 & 2.1 & 82 \\
\hline Trumpler 5 & 9.4 & 17.0 & $0.62 \pm 0.07$ & $40.6 \pm 5.8$ & 39 & 3.17 & $17.6 \pm 0.6$ & 86 & 15.6 & 73 \\
\hline NGC 2301 & 9.2 & 4.5 & $0.47 \pm 0.02$ & $38.2 \pm 7.4$ & 7 & 2.52 & $6.0 \pm 0.5$ & 51 & 3.5 & 73 \\
\hline NGC 2323 & 9.3 & -20.9 & $0.75 \pm 0.05$ & $21.0 \pm 3.5$ & 24 & 4.08 & $6.8 \pm 0.3$ & 74 & 5.1 & 79 \\
\hline NGC 2345 & 9.9 & -78.1 & $1.75 \pm 0.24$ & $15.0 \pm 1.3$ & 51 & 6.42 & $8.2 \pm 0.2$ & 89 & 7.6 & 55 \\
\hline NGC 2354 & 9.8 & -247.3 & $0.86 \pm 0.10$ & $5.1 \pm 0.9$ & 31 & 8.86 & $5.0 \pm 0.1$ & 87 & 4.5 & 89 \\
\hline NGC 2360 & 9.5 & -35.6 & $0.94 \pm 0.07$ & $18.6 \pm 2.6$ & 29 & 5.57 & $6.8 \pm 0.2$ & 76 & 5.4 & 84 \\
\hline NGC 2420 & 10.9 & 826.6 & $1.05 \pm 0.02$ & $16.1 \pm 2.2$ & 7 & 8.40 & $1.9 \pm 0.1$ & 50 & 1.1 & 81 \\
\hline NGC 2421 & 9.8 & 3.5 & $1.37 \pm 0.09$ & $20.1 \pm 1.8$ & 44 & 8.33 & $10.0 \pm 0.2$ & 86 & 8.7 & 84 \\
\hline NGC 2437 & 9.5 & 106.6 & $2.27 \pm 0.13$ & $14.4 \pm 0.9$ & 36 & 9.30 & $6.3 \pm 0.1$ & 96 & 5.2 & 75 \\
\hline NGC 2447 & 9.0 & 3.5 & $0.51 \pm 0.03$ & $31.0 \pm 6.1$ & 23 & 2.83 & $9.1 \pm 0.5$ & 74 & 7.0 & 78 \\
\hline NGC 2477 & 9.2 & -181.5 & $2.16 \pm 0.16$ & $22.3 \pm 1.2$ & 19 & 6.90 & $7.8 \pm 0.2$ & 49 & 4.2 & 76 \\
\hline NGC 2533 & 9.2 & 35.2 & $1.25 \pm 0.20$ & $14.1 \pm 1.7$ & 31 & 5.97 & $5.2 \pm 0.2$ & 80 & 4.4 & 73 \\
\hline NGC 3114 & 8.3 & -59.2 & $0.50 \pm 0.04$ & $35.2 \pm 6.7$ & 14 & 5.73 & $7.3 \pm 0.3$ & 73 & 5.3 & 90 \\
\hline IC 2714 & 8.1 & -35.7 & $1.27 \pm 0.09$ & $43.0 \pm 2.9$ & 44 & 4.69 & $21.9 \pm 0.5$ & 82 & 18.7 & 74 \\
\hline Mel 105 & 7.9 & -88.8 & $1.16 \pm 0.15$ & $26.1 \pm 2.5$ & 45 & 7.13 & $13.1 \pm 0.2$ & 88 & 11.7 & 82 \\
\hline NGC 4755 & 7.6 & 91.8 & $0.63 \pm 0.10$ & $21.1 \pm 3.8$ & 26 & 5.49 & $7.4 \pm 0.3$ & 89 & 6.7 & 77 \\
\hline NGC 5617 & 7.5 & -2.7 & $1.50 \pm 0.26$ & $23.2 \pm 1.8$ & 60 & 4.54 & $16.7 \pm 0.4$ & 81 & 13.8 & 82 \\
\hline NGC 6208 & 7.4 & -126.2 & $0.94 \pm 0.14$ & $12.3 \pm 2.1$ & 38 & 4.14 & $5.7 \pm 0.3$ & 80 & 4.6 & 78 \\
\hline NGC 6250 & 7.6 & -29.6 & $0.74 \pm 0.09$ & $13.8 \pm 2.8$ & 25 & 2.91 & $4.1 \pm 0.3$ & 75 & 3.3 & 62 \\
\hline NGC 6451 & 6.6 & -53.5 & $2.63 \pm 0.51$ & $36.5 \pm 1.3$ & 71 & 6.98 & $28.7 \pm 0.3$ & 90 & 26.1 & 78 \\
\hline NGC 6531 & 7.2 & -9.0 & $0.67 \pm 0.06$ & $16.0 \pm 3.3$ & 26 & 3.07 & $5.2 \pm 0.3$ & 79 & 4.4 & 66 \\
\hline NGC 6546 & 7.6 & -22.6 & $0.98 \pm 0.12$ & $34.7 \pm 3.4$ & 43 & 3.32 & $19.4 \pm 0.6$ & 74 & 14.9 & 80 \\
\hline NGC 6705 & 6.9 & -62.6 & $0.72 \pm 0.10$ & $43.4 \pm 5.2$ & 31 & 5.54 & $15.8 \pm 0.4$ & 82 & 13.2 & 86 \\
\hline NGC 6755 & 7.3 & -46.4 & $2.87 \pm 0.35$ & $31.3 \pm 1.1$ & 58 & & & & 13.0 & \\
\hline NGC 6811 & 8.4 & 266.4 & $0.49 \pm 0.09$ & $21.6 \pm 5.4$ & 19 & 3.77 & $6.3 \pm 0.3$ & 70 & 4.6 & 88 \\
\hline NGC 6819 & 8.2 & 249.7 & $2.22 \pm 0.21$ & $26.2 \pm 1.3$ & 41 & 5.31 & $12.2 \pm 0.3$ & 86 & 10.7 & 25 \\
\hline NGC 6866 & 8.4 & 179.0 & $0.77 \pm 0.06$ & $13.6 \pm 2.7$ & 19 & 4.73 & $3.7 \pm 0.2$ & 63 & 2.5 & 85 \\
\hline NGC 6939 & 8.7 & 256.1 & $0.54 \pm 0.02$ & $58.6 \pm 8.0$ & 9 & 3.89 & $9.4 \pm 0.4$ & 58 & 5.9 & 82 \\
\hline NGC 6940 & 8.2 & -130.6 & $1.67 \pm 0.17$ & $11.4 \pm 1.1$ & 45 & 8.03 & $6.3 \pm 0.2$ & 79 & 5.1 & 86 \\
\hline NGC 7654 & 9.2 & 13.0 & $1.59 \pm 0.08$ & $22.4 \pm 1.7$ & 27 & 4.48 & $8.4 \pm 0.3$ & 65 & 6.1 & 66 \\
\hline
\end{tabular}

The histogram of cluster members population in corona is shown in Fig. 2d. It peaks around $80 \%$. This clearly indicates the importance of the corona in studies dealing with entire stellar contents of the cluster e.g., study of mass function. On the other hand, for the determination of cluster parameters like distance and age, stars of the cluster core are best suited as they have less field star contamination. The cluster sequences in photometric diagrams can therefore be better identified in stars of the core region.

\subsection{Correlations between parameters}

Figure 3 shows correlation of the core size with the corona size, the cluster members density and the percentage of field star contamination $\left(F_{0}\right)$ in the core. It indicates that both the corona size and $F_{0}$ value generally increase while the core density decreases with increasing size of the core. At a given core size, the corona size varies by about 3 to $5 \mathrm{pc}$; the core density varies from few to few tens of star $/ \mathrm{pc}^{2}$ while the $F_{0}$ value changes from few tens to $50 \%$. 
Table 3. Exponential powers of the variation of stellar surface density $(\rho)$ with radius $(R)$ in relations $\rho \propto \mathrm{e}^{-B R}$ and $\rho \propto R^{-\gamma}$ up to the core and cluster boundaries. $c_{1}$ and $c_{2}$ are correlation coefficients of least-square solutions of the linear relations used to derive the values of $B, \sigma_{B}$ and $\gamma, \sigma_{\text {gamma }}$ respectively.

\begin{tabular}{|c|c|c|c|c|c|c|c|c|}
\hline \multirow[b]{2}{*}{ Name } & \multicolumn{4}{|c|}{ Core region } & \multicolumn{4}{|c|}{ Core plus corona region } \\
\hline & $B \pm \sigma_{B}$ & $c_{1}$ & $\gamma \pm \sigma_{\gamma}$ & $c_{2}$ & $B \pm \sigma_{B}$ & $c_{1}$ & $\gamma \pm \sigma_{\gamma}$ & $c_{2}$ \\
\hline NGC 188 & $0.002 \pm 0.0005$ & 0.94 & $0.32 \pm 0.07$ & 0.93 & $0.003 \pm 0.0002$ & 0.98 & $0.86 \pm 0.14$ & 0.89 \\
\hline NGC 654 & $0.006 \pm 0.0016$ & 0.97 & $0.49 \pm 0.25$ & 0.89 & $0.003 \pm 0.0005$ & 0.90 & $0.63 \pm 0.07$ & 0.96 \\
\hline NGC 663 & $0.001 \pm 0.0005$ & 0.88 & $0.13 \pm 0.05$ & 0.88 & $0.001 \pm 0.0002$ & 0.92 & $0.35 \pm 0.05$ & 0.93 \\
\hline NGC 1245 & $0.004 \pm 0.0002$ & 1.00 & $0.33 \pm 0.05$ & 0.99 & $0.002 \pm 0.0003$ & 0.92 & $0.59 \pm 0.05$ & 0.97 \\
\hline NGC 1817 & $0.010 \pm 0.0001$ & 1.00 & $0.67 \pm 0.01$ & 1.00 & $0.001 \pm 0.0002$ & 0.84 & $0.33 \pm 0.04$ & 0.91 \\
\hline NGC 1960 & $0.004 \pm 0.0014$ & 0.90 & $0.41 \pm 0.22$ & 0.79 & $0.002 \pm 0.0003$ & 0.93 & $0.67 \pm 0.07$ & 0.95 \\
\hline NGC 2099 & $0.003 \pm 0.0005$ & 0.95 & $0.33 \pm 0.10$ & 0.89 & $0.001 \pm 0.0001$ & 0.93 & $0.56 \pm 0.04$ & 0.96 \\
\hline NGC 2244 & $0.002 \pm 0.0008$ & 0.78 & $0.26 \pm 0.06$ & 0.93 & $0.001 \pm 0.0001$ & 0.93 & $0.35 \pm 0.04$ & 0.91 \\
\hline Trumpler 5 & $0.003 \pm 0.0025$ & 0.79 & $0.32 \pm 0.16$ & 0.90 & $0.002 \pm 0.0002$ & 0.92 & $0.45 \pm 0.03$ & 0.97 \\
\hline NGC 2301 & $0.009 \pm 0.0026$ & 0.96 & $0.72 \pm 0.39$ & 0.88 & $0.003 \pm 0.0005$ & 0.92 & $0.90 \pm 0.08$ & 0.96 \\
\hline NGC 2323 & $0.005 \pm 0.0002$ & 1.00 & $0.44 \pm 0.07$ & 0.99 & $0.002 \pm 0.0002$ & 0.91 & $0.57 \pm 0.04$ & 0.98 \\
\hline NGC 2345 & $0.002 \pm 0.0006$ & 0.95 & $0.22 \pm 0.10$ & 0.83 & $0.001 \pm 0.0002$ & 0.90 & $0.31 \pm 0.04$ & 0.92 \\
\hline NGC 2354 & $0.008 \pm 0.0001$ & 1.00 & $0.55 \pm 0.01$ & 1.00 & $0.001 \pm 0.0002$ & 0.82 & $.35 \pm 0.03$ & 0.97 \\
\hline NGC 2360 & $0.005 \pm 0.0001$ & 1.00 & $0.44 \pm 0.09$ & 0.98 & $0.001 \pm 0.0002$ & 0.89 & $0.46 \pm 0.04$ & 0.97 \\
\hline NGC 2420 & $0.010 \pm 0.0001$ & 1.00 & $0.64 \pm 0.01$ & 1.00 & $0.003 \pm 0.0006$ & 0.90 & $0.98 \pm 0.07$ & 0.97 \\
\hline NGC 2421 & $0.004 \pm 0.0007$ & 0.98 & $0.32 \pm 0.01$ & 1.00 & $0.001 \pm 0.0001$ & 0.88 & $0.33 \pm 0.01$ & 0.99 \\
\hline NGC 2437 & $0.002 \pm 0$ & 0.98 & $0.24 \pm 0.03$ & 0.97 & $0.001 \pm 0.0001$ & 0.93 & $0.43 \pm 0.03$ & 0.96 \\
\hline NGC 2447 & $0.002 \pm 0.0003$ & 0.93 & $0.59 \pm 0.03$ & 0.99 & $0.002 \pm 0.0003$ & 0.93 & $0.59 \pm 0.03$ & 0.99 \\
\hline NGC 2477 & $0.004 \pm 0.0001$ & 1.00 & $0.27 \pm 0.01$ & 1.00 & $0.002 \pm 0.0001$ & 0.99 & $0.66 \pm 0.06$ & 0.94 \\
\hline NGC 2533 & $0.004 \pm 0.0035$ & 0.76 & $0.29 \pm 0.38$ & 0.61 & $0.001 \pm 0.0002$ & 0.84 & $0.41 \pm 0.05$ & 0.93 \\
\hline NGC 3114 & $0.009 \pm 0.0011$ & 0.99 & $0.72 \pm 0.25$ & 0.95 & $0.001 \pm 0.0001$ & 0.89 & $0.58 \pm 0.04$ & 0.96 \\
\hline IC 2714 & $0.002 \pm 0.0004$ & 0.95 & $0.21 \pm 0.05$ & 0.95 & $0.001 \pm 0.0001$ & 0.91 & $0.36 \pm 0.03$ & 0.96 \\
\hline Mel 105 & $0.004 \pm 0.0013$ & 0.95 & $0.36 \pm 0.04$ & 0.99 & $0.001 \pm 0.0001$ & 0.92 & $0.33 \pm 0.03$ & 0.97 \\
\hline NGC 4755 & $0.008 \pm 0.0001$ & 1.00 & $0.52 \pm 0.01$ & 1.00 & $0.002 \pm 0.0004$ & 0.85 & $0.44 \pm 0.05$ & 0.96 \\
\hline NGC 5617 & $0.002 \pm 0.0007$ & 0.91 & $0.26 \pm 0.04$ & 0.98 & $0.001 \pm 0.0001$ & 0.90 & $0.25 \pm 0.02$ & 0.95 \\
\hline NGC 6208 & $0.005 \pm 0.0002$ & 1.00 & $0.45 \pm 0.08$ & 0.99 & $0.002 \pm 0.0002$ & 0.91 & $0.46 \pm 0.06$ & 0.92 \\
\hline NGC 6250 & $0.004 \pm 0.0022$ & 0.87 & $0.28 \pm 0.25$ & 0.74 & $0.002 \pm 0.0003$ & 0.88 & $0.60 \pm 0.08$ & 0.92 \\
\hline NGC 6451 & $0.001 \pm 0.0004$ & 0.85 & $0.12 \pm 0.08$ & 0.67 & $0.001 \pm 0.0001$ & 0.84 & $0.13 \pm 0.02$ & 0.87 \\
\hline NGC 6531 & $0.007 \pm 0.0001$ & 1.00 & $0.44 \pm 0.01$ & 1.00 & $0.003 \pm 0.0006$ & 0.87 & $0.72 \pm 0.11$ & 0.92 \\
\hline NGC 6546 & $0.002 \pm 0.0011$ & 0.83 & $0.25 \pm 0.10$ & 0.88 & $0.001 \pm 0.0002$ & 0.89 & $0.37 \pm 0.04$ & 0.94 \\
\hline NGC 6705 & $0.009 \pm 0.0001$ & 1.00 & $0.58 \pm 0.01$ & 1.00 & $0.001 \pm 0.0002$ & 0.88 & $0.40 \pm 0.03$ & 0.97 \\
\hline NGC 6755 & $0.002 \pm 0.0008$ & 0.81 & $0.34 \pm 0.10$ & 0.86 & & & & \\
\hline NGC 6811 & $0.009 \pm 0.0001$ & 1.00 & $0.59 \pm 0.01$ & 1.00 & $0.001 \pm 0.0002$ & 0.83 & $0.49 \pm 0.08$ & 0.91 \\
\hline NGC 6819 & $0.002 \pm 0.0007$ & 0.89 & $0.29 \pm 0.16$ & 0.79 & $0.001 \pm 0.0001$ & 0.92 & $0.45 \pm 0.04$ & 0.95 \\
\hline NGC 6866 & $0.007 \pm 0.0001$ & 1.00 & $0.48 \pm 0.01$ & 1.00 & $0.002 \pm 0.0003$ & 0.91 & $0.61 \pm 0.05$ & 0.98 \\
\hline NGC 6939 & $0.009 \pm 0.0001$ & 1.00 & $0.60 \pm 0.01$ & 1.00 & $0.003 \pm 0.0004$ & 0.93 & $0.82 \pm 0.02$ & 1.00 \\
\hline NGC 6940 & $0.001 \pm 0.0021$ & 0.33 & $0.34 \pm 0.29$ & 0.50 & $0.001 \pm 0.0001$ & 0.73 & $0.33 \pm 0.05$ & 0.78 \\
\hline NGC 7654 & $0.003 \pm 0.0003$ & 0.99 & $0.27 \pm 0.08$ & 0.92 & $0.002 \pm 0.0002$ & 0.95 & $0.55 \pm 0.06$ & 0.94 \\
\hline
\end{tabular}

The scatter in Figs. 3a and $\mathrm{c}$ is almost independent of the core size. However, the scatter in the density generally decreases as the core size increases, being largest for a core size of around $0.6 \mathrm{pc}$. A larger sample is needed to ascertain the behaviour.

In order to study correlation of the corona size $\left(R_{\mathrm{cn}}-\right.$ $\left.R_{0}\right)$ with its cluster member density $\left(\rho_{\mathrm{cn}}-\rho_{\mathrm{f}}\right)$, field star contamination $\left(F_{\mathrm{cn}}\right)$ and fraction of cluster members $\left(M_{\mathrm{cn}}\right)$, we plot them in Fig. 4 . The mean value of members' density in the coronal region is $1.6 \pm 0.99 \mathrm{star} / \mathrm{pc}^{2}$. It slowly decreases with inceasing corona size and the value becomes $0.9 \pm 0.4 \mathrm{star} / \mathrm{pc}^{2}$ for a corona size larger than 8 pc (see Fig. 4c). Both the $F_{\mathrm{cn}}$ and $M_{\mathrm{cn}}$ values are generally more than $60 \%$ with no statistically significant dependence on the corona size (see Figs. $4 \mathrm{a}$ and b).

\section{Effect of galactic location on cluster parameters}

The open star clusters form from molecular clouds having mass in the range of several thousands to hundreds of thousands of $M_{\odot}$. As the Galactic tidal forces are different at different locations, it may be worthwhile to see how they affect the sizes and member densities in open star clusters. For this, we study the variation of cluster parameters with 

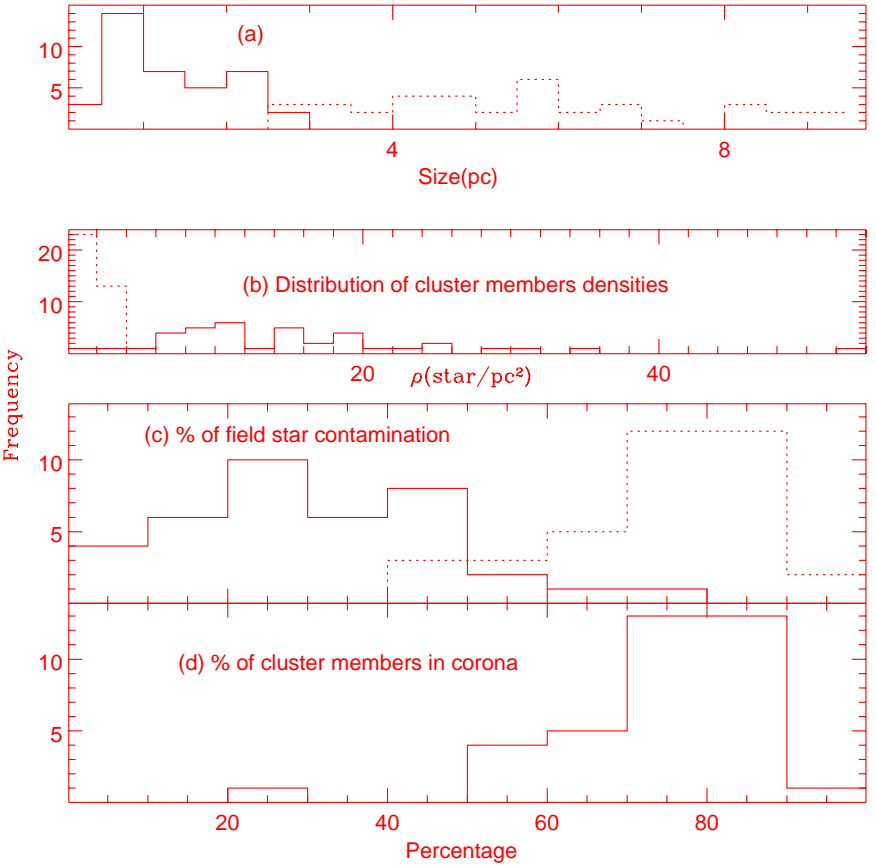

Fig. 2. Histograms of the core $\left(R_{0}\right)$ and corona $\left(R_{\mathrm{cn}}-R_{0}\right)$ sizes; their densities of cluster members and field star contamination along with fraction of cluster members in corona of clusters under study are presented in $\mathbf{a}-\mathbf{d}$ ) respectively. The solid and dashed lines in $\mathbf{a}-\mathbf{c}$ ) denote core and coronal regions respectively.

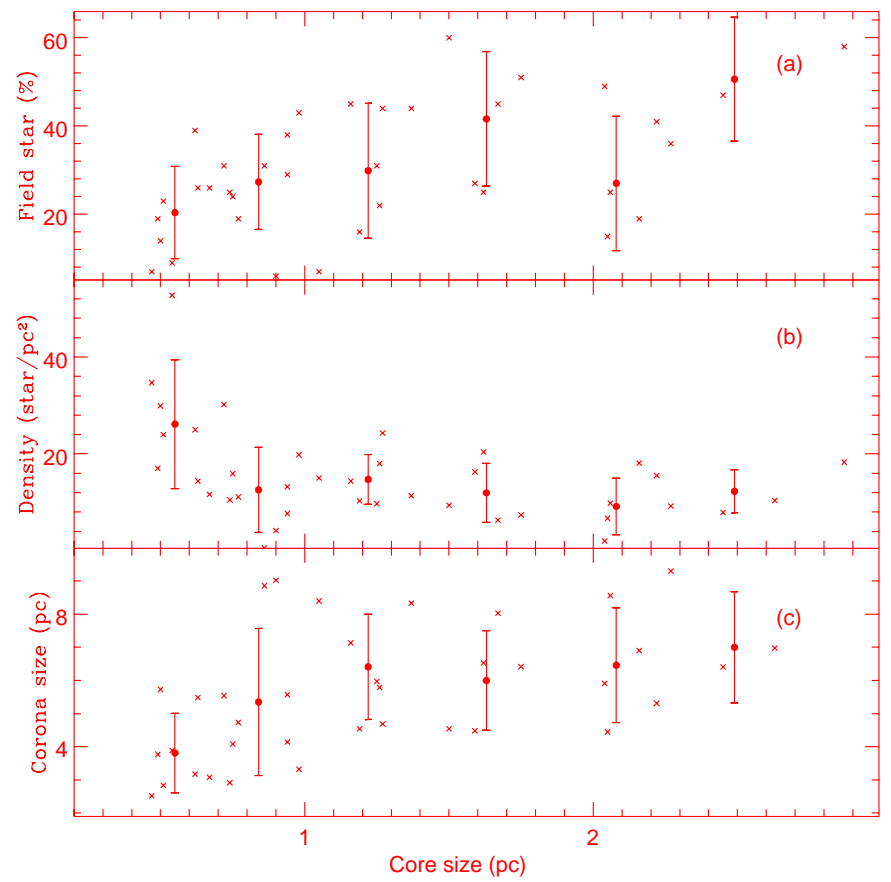

Fig. 3. The corona size $\left(R_{\mathrm{cn}}-R_{0}\right)$, the cluster member density $\left(\rho_{0}-\rho_{\mathrm{f}}\right)$ in the core and the percentage of field star contamination $\left(F_{0}\right)$ are plotted as crosses against the core size in $\mathbf{c}$ ), b) and a) respectively. Binned data points along with their $\sigma$ are shown by filled circles and error bars respectively.

the Galacto-centric distance $\left(R_{\mathrm{G}}\right)$ and height $(z)$ above the Galactic plane in the following sub-sections.

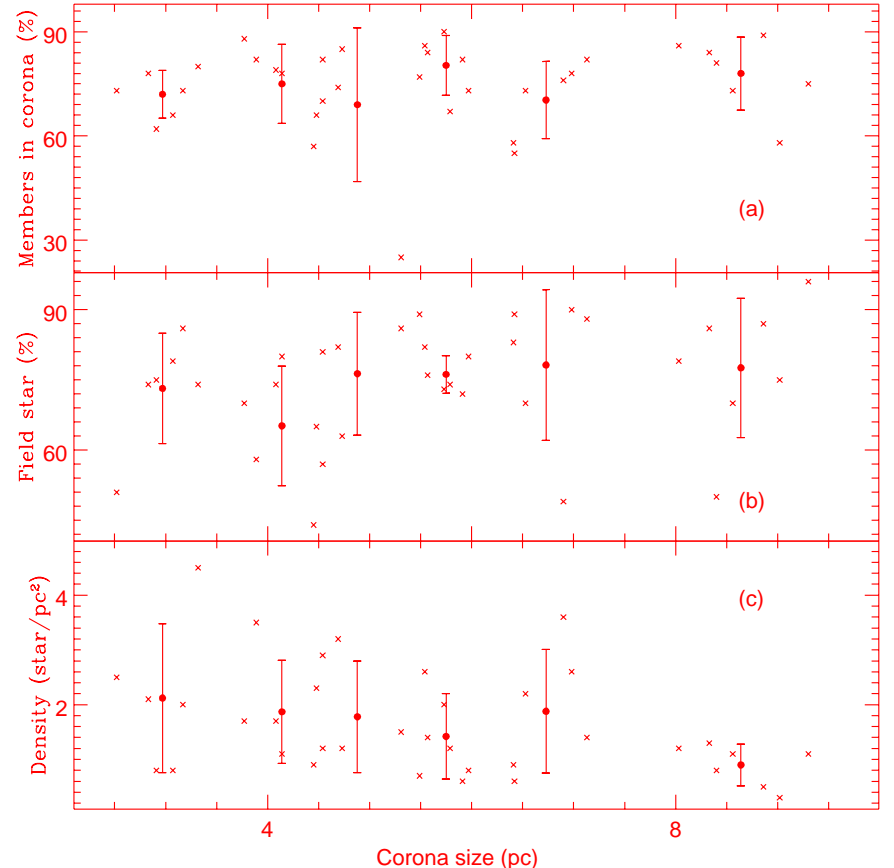

Fig. 4. The cluster member density $\left(\rho_{\mathrm{cn}}-\rho_{\mathrm{f}}\right)$, percentage of the field stars $\left(F_{\mathrm{cn}}\right)$ and the cluster members in coronal regions $\left(M_{\mathrm{cn}}\right)$ are plotted as crosses against the corona size $\left(R_{\mathrm{cn}}-\right.$ $\left.R_{0}\right)$ in c), b) and a) respectively. Filled circles and error bars denote the same as in Fig. 3.

\subsection{Dependence of cluster parameters on the $R_{\mathrm{G}}$}

We plot the core and corona sizes, the central concentration and the member densities ratio of the core to corona against the $R_{\mathrm{G}}$ in Figs. 5a-d respectively. Though the errors are large, it illustrates that the mean core size is independent of $R_{\mathrm{G}}$. Similarly, both the ratio of corona to core sizes and the $\left(\frac{\rho_{0}-\rho_{\mathrm{f}}}{\rho_{\mathrm{cn}}-\rho_{\mathrm{f}}}\right)$ values seem to have no statistically significant dependence on $R_{\mathrm{G}}$. On the other hand, the mean corona size of $\sim 5 \mathrm{pc}$ for $R_{\mathrm{G}}<9.5 \mathrm{kpc}$ increases systematically with $R_{\mathrm{G}}$ thereafter and becomes $\sim 7.1 \mathrm{pc}$ at $R_{\mathrm{G}}=10.5 \mathrm{kpc}$. A careful inspection of the data reveals that the observed correlations show no dependence on cluster ages.

\subsection{Dependence of cluster parameters on the $z$}

Figure 6 outlines the plot of sizes and cluster member densities of both the core and corona of the cluster against its height $(z)$ above the Galactic plane. None of the parameters seem to depend on the value of $z$. As expected, most of the clusters with $-200 \mathrm{pc}<z<200 \mathrm{pc}$ are younger than 100 Myr while those with $z<-200 \mathrm{pc}$ and $z>$ $200 \mathrm{pc}$ are older than that. We therefore conclude that irrespective of the core and corona sizes, the clusters older than 100 Myr are generally located away from while the younger ones are confined to the Galactic plane. 


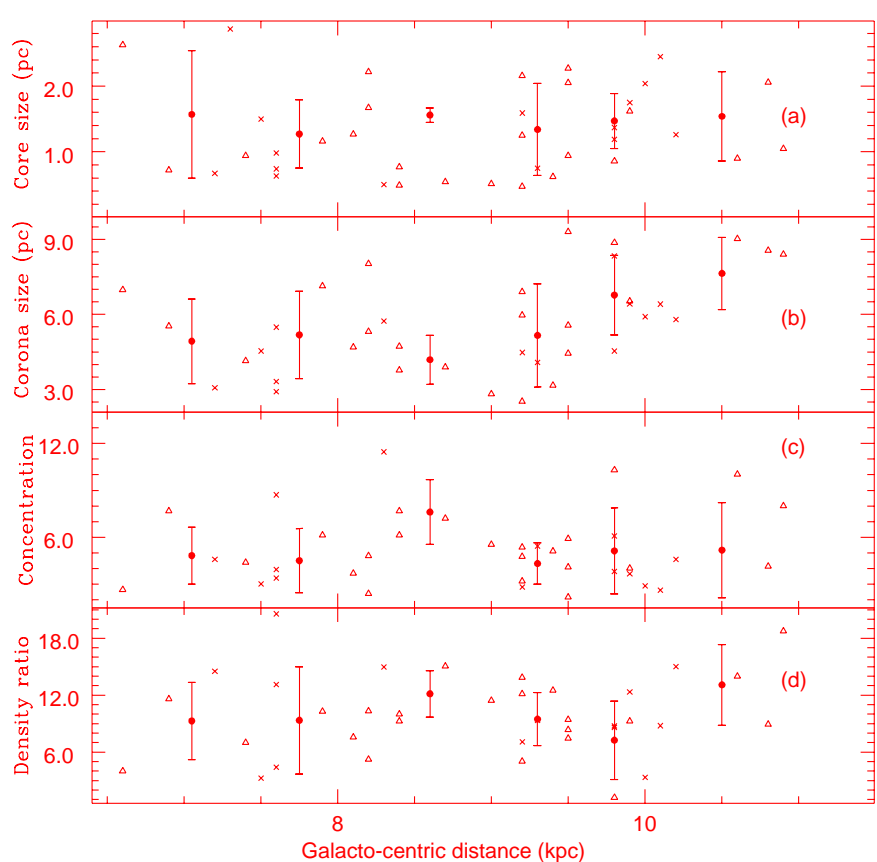

Fig. 5. Plot of the core and coronal sizes; the central concentration defined as ratio of the corona to core sizes and the members density ratios of core to corona against $R_{\mathrm{G}}$ are shown in a-d) respectively. In order to study the trend in variation, data points have been binned in $R_{\mathrm{G}}$. Error bars are the $\sigma$ of data points in a bin. Crosses denote clusters younger than $100 \mathrm{Myr}$ while olders are shown by triangles.

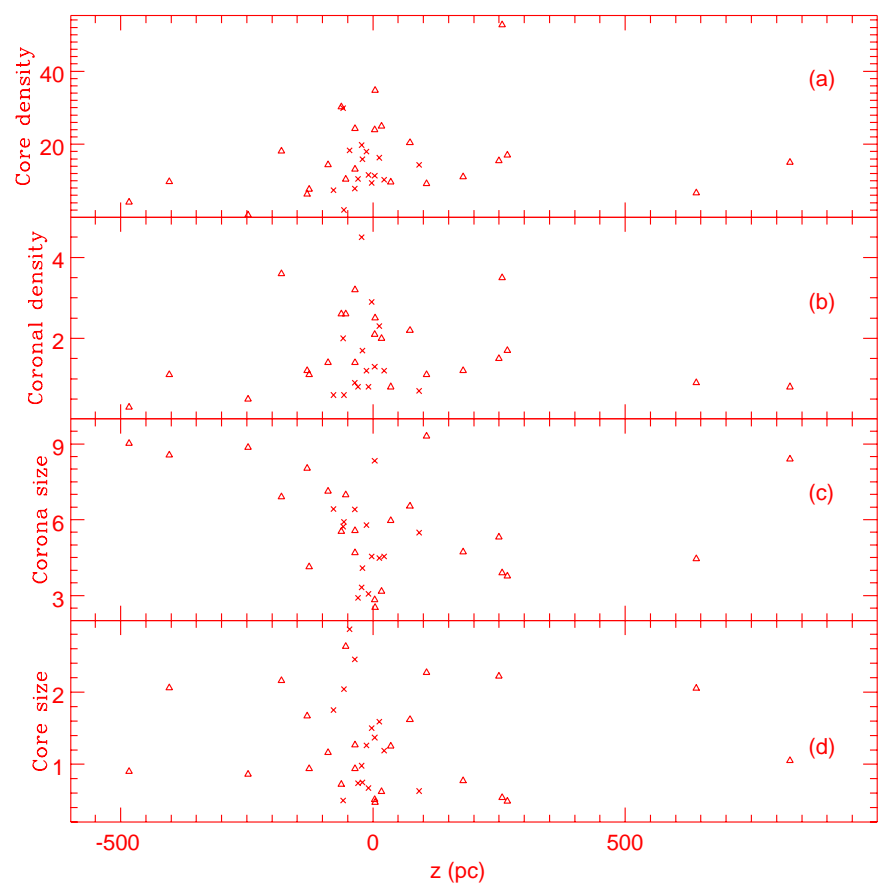

Fig. 6. Plot of sizes and densities of both the core and coronal regions of the cluster against $z$, its location above or below the Galactic plane. Crosses denote clusters younger than $100 \mathrm{Myr}$ while olders are shown by triangles.

\section{Effect of dynamical evolution on cluster parameters}

Stellar encounters in a cluster can change the spatial position of members with time due to equi-partition of

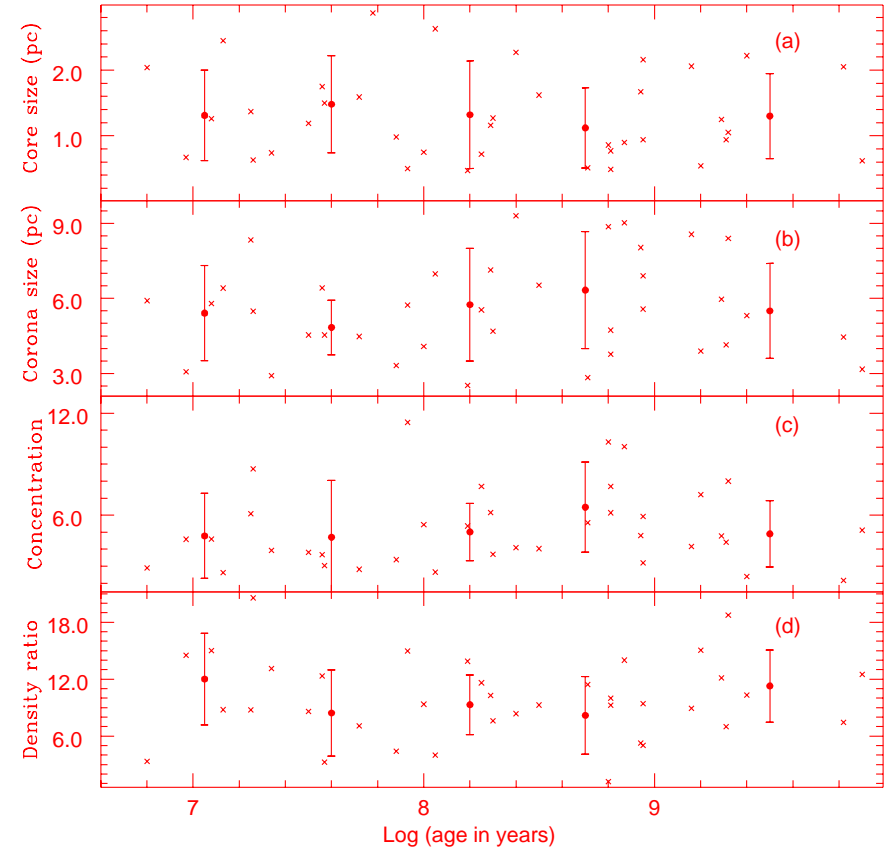

Fig. 7. The core and corona sizes; central concentration and density ratio of core to corona are plotted against log of age in a-d) respectively. Filled circles and error bars show the mean and $\sigma$ respectively of the data points binned in log (age).

energies amongst the cluster members. In order to see its effect on parameters of open star clusters, we the plot core and corona sizes, central concentration and density ratio of core to corona against log (age) in Figs. 7a-d respectively. We observe no statistically significant trend at all in the sizes of core and corona as well as in the ratio of their cluster member densities with age. However, central concentration appears to be somewhat smaller for clusters older than 1000 Myr.

\section{Discussions and conclusions}

The present study of the spatial structures of 38 rich galactic open star clusters leads to the following useful conclusions:

1. On average, annular size of the coronal region is about 5 times the core radius. All the clusters including the very young ones have a corona. Presence of a core and corona have also been detected in NGC 6611, an extremely young (few Myr) star cluster by Belikov et al. (1999, 2000) using proper motion data. As the RDP of the cluster (core plus corona) region can be fitted by a function of $\exp (-B R)$ or $R^{-\gamma}$ types, we consider the coronal region as an integral outer part of a cluster existing from the time of its formation. If the appearance of a corona is only due to the dynamical evolution in the cluster, it should appear only for those clusters that are older than $\sim 100$ Myr. 
2. Field star contamination in the coronal region, on average, is $44 \%$ greater to that of the core region. To determine the cluster parameters from the observed colourmagnitude and colour-colour diagrams, stars located in the core region should be considered. However, for the studies dealing with the entire stellar content of the cluster, members present in the coronal region must be included as they form a large fraction of cluster members. This clearly demonstrates the importance of coronal regions in the studies of IMF and dynamical evolution of the clusters.

3. In contrast to the coronal regions, the structure of the cluster cores does not vary significantly from one cluster to other.

4. The mean radii of the core and cluster sizes are $1.3 \pm 0.67$ and $7.0 \pm 2.4$ pc respectively while the corresponding median values are 1.2 and 6.8 pc respectively. The mean values of cluster diameters determined by both Lyngå (1982) and Janes \& Phelps (1994) are $\sim 5.3$ pc which lie between the values of core and cluster diameter determined by us. Present values should be considered more reliable, as they are derived from the RDP of an extended region around the cluster.

5. We find that the mean size of the clusters with $R_{\mathrm{G}}<$ $9.5 \mathrm{kpc}$ is smaller than that of those with $R_{\mathrm{G}}>$ $9.5 \mathrm{kpc}$. We thus confirm the earlier findings of Burki \& Maeder (1976), Lyngå (1982) and Janes et al. (1988).

6. In agreement with Lyngå (1982) and Janes et al. (1988), we find that sizes of the clusters are independent of age. Hence we believe that the cluster size may depend on the properties of the parental molecular cloud rather than the cluster age.

\section{Appendix}

USNO-A V2.0: A catalogue of astrometric standards

USNO-A2.0 is a catalogue of 526280881 stars, and is based on a re-reduction of the Precision Measuring Machine (PMM) scans for astrometric and photometric data. These stars were detected by the PMM, built and operated by the U. S. Naval Observatory, Flagstaff Station during the scanning and processing of the Palomar Observatory Sky Survey I (POSS-I) O and E plates, the UK Science and Research Council SRC- $J$ survey plates, and the European Southern Observatory ESO- $R$ survey plates. For field centers with $\delta<-30$ degrees, the data come from POSS- $I$ plates, while the data for the remaining part of the sky come from
SRC- $J$ and ESO- $R$ plates. The errors arising from the PMM are in the range of 0.15 arcsec in astrometry and $0.15 \mathrm{mag}$ in photometry. To avoid the necessity of consulting many catalogues, objects brighter than 11th magnitude that appear in the Guide Star Catalogue that were not detected by the PMM were inserted.

Acknowledgements. The valuable suggestions provided by the referee Dr. B. Chen improved the scientific content of the paper. We are thankful to Dr. A. E. Piskunov for useful discussions and to $\mathrm{B}$. Kumar for reading the manuscript critically. The present research has used the USNO-A V2.0 catalogue and the WEBDA open cluster data base Web site at http://obswww.unige.ch/webda/ maintained by Dr. J. C. Mermilliod.

\section{References}

Artyukhina, N. M., \& Kholopov, P. N. 1959, SvA-AJ, 3, 923 Artyukhina, N. M., \& Kholopov, P. N. 1963, SvA-AJ, 7, 840 Artyukhina, N. M., \& Kholopov, P. N. 1966, SvA-AJ, 10, 448 Artyukhina, N. M. 1966, SvA-AJ, 10, 103

Belikov, A. N., Kharchenko, N. V., Piskunov, A. E., \& Schilbach, E. 1999, A\&AS, 134, 525

Belikov, A. N., Kharchenko, N. V., Piskunov, A. E., \& Schilbach, E. 2000, A\&A, 358, 886

Burki, G., \& Maeder, A. 1976, A\&A, 51, 247

Durgapal, A. K., \& Pandey, A. K. 2001, A\&A, 375, 504

Elmegreen, B. G., \& Efremov, Yu. N. 1997, ApJ, 480, 235

Janes, K. A., Tilley, C., \& Lyngå, G. 1988, AJ, 95, 771

Janes, K. A., \& Phelps, R. L. 1994, AJ, 108, 1773

Kaluzny, J. 1992, Acta Astron., 42, 29

Kholopov, P. N. 1969, SvA-AJ, 12, 625

Loktin, A. V., Matkin, N. V., \& Gerasimenko, T. P. 1994, Astron. Astrophys. Trans., 4, 153

Lyngå, G. 1982, A\&A, 109, 213

Lyngå, G. 1987, in Catalog of Open Cluster Data, Computer Based Catalogue available through the CDS, Starsbourg, France and through NASA Data Center, GreenBelt, MaryLand, USA, 5th edition, 4, 121

Mathieu, R. D. 1985, IAU Symp., 113, 427

Monet, D., Bird, A., Canzian, A., et al. 1998, USNO-A2.0, (U.S. Naval Observatory, Washington DC)

Nilakshi, \& Sagar, R. 2002, A\&A, 381, 65

Pandey, A. K., Nilakshi, Ogura, K., Sagar, R., \& Tarusawa, K. 2001, A\&A, 374, 504

Sagar, R., \& Griffiths, W. K. 1998, MNRAS, 299, 777

Salpeter, E. E. 1955, ApJ, 121, 161

Shapley, H. 1916, Contr. Mt. Wilson Obs., 117

Subramaniam, A., Gorti, U., Sagar, R., \& Bhatt, H. C. 1995, A\&A, 302, 86

Turner, D. G. 1982, PASP, 94, 1003

Wilner, D. J., \& Lada, C. J. 1991, AJ, 102, 1050 